Georgian Mathematical Journal

Volume 13 (2006), Number 3, 433-445

\title{
GROUND STATE SOLUTIONS OF NONLINEAR STATIONARY SCHRÖDINGER SYSTEMS WITH DISCONTINUOUS NONLINEARITY AND VARIABLE POTENTIAL
}

\author{
TEODORA-LILIANA DINU
}

\begin{abstract}
We establish the existence of an entire solution for a class of stationary Schrödinger systems with subcritical discontinuous nonlinearities and lower bounded potentials that blow-up at infinity. The proof is based on the critical point theory in the sense of Clarke and we apply the Mountain Pass Lemma for locally Lipschitz functionals. Our result generalizes in a nonsmooth framework a result of Rabinowitz [16] on the existence of entire solutions of the nonlinear Schrödinger equation.
\end{abstract}

2000 Mathematics Subject Classification: 35J50, 49J52, 58E05.

Key words and phrases: Nonlinear elliptic system, entire solution, Lipschitz functional, Clarke generalized gradient.

\section{Introduction And the Main Result}

The Schrödinger equation plays the role of Newton's laws and conservation of energy in classical mechanics, that is, it predicts the future behaviour of a dynamic system. The linear form of Schrödinger's equation is

$$
\Delta \psi+\frac{8 \pi^{2} m}{\hbar^{2}}(W(x)-V(x)) \psi=0,
$$

where $\psi$ is the Schrödinger wave function, $m$ is the mass, $\hbar$ denotes Planck's constant, $W$ is the energy, and $V$ stands for the potential energy. The structure of the nonlinear Schrödinger equation is much more complicated. This equation describes various phenomena arising in: self-channelling of a high-power ultrashort laser in matter, in the theory of Heisenberg ferromagnets and magnons, in dissipative quantum mechanics, in condensed matter theory, in plasma physics (e.g., the Kurihara superfluid film equation). We refer to $[9,18]$ for a modern overview, including applications.

Consider the model problem

$$
i \hbar \psi_{t}=-\frac{\hbar^{2}}{2 m} \Delta \psi+V(x) \psi-\gamma|\psi|^{p-1} \psi \quad \text { in } \mathbb{R}^{N}(N \geq 2),
$$

where $p<2 N /(N-2)$ if $N \geq 3$ and $p<+\infty$ if $N=2$. In the study of this equation $\mathrm{Oh}$ [15] supposed that the potential $V$ is bounded and possesses a non-degenerate critical point at $x=0$. More precisely, it is assumed that $V$ belongs to the class $\left(V_{a}\right)$ (for some $a$ ) introduced in Kato [13]. Taking $\gamma>0$ and $\hbar>0$ sufficiently small and using a Lyapunov-Schmidt type reduction, Oh 
[15] proved the existence of a standing wave solution of Problem (1), that is, a solution of the form

$$
\psi(x, t)=e^{-i W t / \hbar} u(x) .
$$

Note that substituting the ansatz (2) into (1) leads to

$$
-\frac{\hbar^{2}}{2} \Delta u+(V(x)-W) u=|u|^{p-1} u \text {. }
$$

The change of variable $y=\hbar^{-1} x$ (and replacing $y$ by $x$ ) yields

$$
-\Delta u+2\left(V_{\hbar}(x)-W\right) u=|u|^{p-1} u \quad \text { in } \mathbb{R}^{N},
$$

where $V_{\hbar}(x)=V(\hbar x)$.

In a celebrated paper, Rabinowitz [16] continued the study of standing wave solutions of nonlinear Schrödinger equations. After making a standing wave ansatz, Rabinowitz reduces the problem to that of studying the semilinear elliptic equation

$$
-\Delta u+b(x) u=f(x, u) \quad \text { in } \mathbb{R}^{N},
$$

under suitable conditions on $b$ and assuming that $f$ is smooth, superlinear and subcritical.

Inspired by Rabinowitz' paper, we consider the following class of coupled elliptic systems in $\mathbb{R}^{N}(N \geq 3)$ :

$$
\begin{cases}-\Delta u_{1}+a(x) u_{1}=f\left(x, u_{1}, u_{2}\right) & \text { in } \mathbb{R}^{N} \\ -\Delta u_{2}+b(x) u_{2}=g\left(x, u_{1}, u_{2}\right) & \text { in } \mathbb{R}^{N}\end{cases}
$$

We point out that coupled nonlinear Schrödinger systems describe some physical phenomena such as the propagation in birefringent optical fibers or Kerr-like photorefractive media in optics. Another motivation to the study of coupled Schrödinger systems arises from the Hartree-Fock theory for the double condensate, that is a binary mixture of Bose-Einstein condensates in two different hyperfine states, cf. [6]. System (3) is also important for industrial applications in fiber communications systems [11] and all-optical switching devices [12]. For important abstract results in Sobolev spaces with applications to partial differential equations we refer to the excellent monographs by Gilbarg and Trudinger [8], and by Hyers, Isac and Rassias [10].

Throughout this paper we assume that $a, b \in L_{\text {loc }}^{\infty}\left(\mathbb{R}^{N}\right)$ and there exist $\underline{a}, \underline{b}>0$ such that $a(x) \geq \underline{a}, \quad b(x) \geq \underline{b}$ a.e. in $\mathbb{R}^{N}$, and $\operatorname{esslim}_{|x| \rightarrow \infty} a(x)=$ $\operatorname{esslim}_{|x| \rightarrow \infty} b(x)=+\infty$. Our aim in this paper is to study the existence of solutions to the above problem in the case when $f, g$ are not continuous functions. Our goal is to show how variational methods can be used to find existence results for stationary nonsmooth Schrödinger systems.

Throughout this paper we assume that $f(x, \cdot, \cdot), g(x, \cdot, \cdot) \in L_{\mathrm{loc}}^{\infty}\left(\mathbb{R}^{2}\right)$. Denote:

$$
\begin{aligned}
& \underline{f}\left(x, t_{1}, t_{2}\right)=\lim _{\delta \rightarrow 0} \operatorname{essinf}\left\{f\left(x, s_{1}, s_{2}\right) ;\left|t_{i}-s_{i}\right| \leq \delta ; i=1,2\right\}, \\
& \bar{f}\left(x, t_{1}, t_{2}\right)=\lim _{\delta \rightarrow 0} \operatorname{esssup}\left\{f\left(x, s_{1}, s_{2}\right) ;\left|t_{i}-s_{i}\right| \leq \delta ; i=1,2\right\}, \\
& \underline{g}\left(x, t_{1}, t_{2}\right)=\lim _{\delta \rightarrow 0} \operatorname{essinf}\left\{g\left(x, s_{1}, s_{2}\right) ;\left|t_{i}-s_{i}\right| \leq \delta ; i=1,2\right\},
\end{aligned}
$$




$$
\bar{g}\left(x, t_{1}, t_{2}\right)=\lim _{\delta \rightarrow 0} \operatorname{esssup}\left\{g\left(x, s_{1}, s_{2}\right) ;\left|t_{i}-s_{i}\right| \leq \delta ; i=1,2\right\} .
$$

Under these conditions we reformulate Problem (3) as follows:

$$
\begin{cases}-\Delta u_{1}+a(x) u_{1} \in\left[\underline{f}\left(x, u_{1}(x), u_{2}(x)\right), \bar{f}\left(x, u_{1}(x), u_{2}(x)\right)\right] & \text { a.e. } x \in \mathbb{R}^{N}, \\ -\Delta u_{2}+b(x) u_{2} \in\left[\underline{g}\left(x, u_{1}(x), u_{2}(x)\right), \bar{g}\left(x, u_{1}(x), u_{2}(x)\right)\right] & \text { a.e. } x \in \mathbb{R}^{N} .\end{cases}
$$

We point out that the corresponding multivalued equation has been considered by Gazzola and Rădulescu in [7].

Let $H^{1}=H\left(\mathbb{R}^{N}, \mathbb{R}^{2}\right)$ denote the Sobolev space of all $U=\left(u_{1}, u_{2}\right) \in\left(L^{2}\left(\mathbb{R}^{N}\right)\right)^{2}$ with weak derivatives $\frac{\partial u_{1}}{\partial x_{j}}, \frac{\partial u_{2}}{\partial x_{j}}(j=1, \ldots, N)$ also in $L^{2}\left(\mathbb{R}^{N}\right)$, endowed with the usual norm

$$
\|U\|_{H_{1}}^{2}=\int_{\mathbb{R}^{N}}\left(|\nabla U|^{2}+|U|^{2}\right) d x=\int_{\mathbb{R}^{N}}\left(\left|\nabla u_{1}\right|^{2}+\left|\nabla u_{2}\right|^{2}+u_{1}^{2}+u_{2}^{2}\right) d x .
$$

Given the functions $a, b: \mathbb{R}^{N} \rightarrow \mathbb{R}$ as above, define the subspace

$$
E=\left\{U=\left(u_{1}, u_{2}\right) \in H^{1} ; \int_{\mathbb{R}^{N}}\left(\left|\nabla u_{1}\right|^{2}+\left|\nabla u_{2}\right|^{2}+a(x) u_{1}^{2}+b(x) u_{2}^{2}\right) d x<+\infty\right\} .
$$

Then the space $E$ endowed with the norm

$$
\|U\|_{E}^{2}=\int_{\mathbb{R}^{N}}\left(\left|\nabla u_{1}\right|^{2}+\left|\nabla u_{2}\right|^{2}+a(x) u_{1}^{2}+b(x) u_{2}^{2}\right) d x
$$

becomes a Hilbert space.

Since $a(x) \geq \underline{a}>0, b(x) \geq \underline{b}>0$, we have the continuous embeddings $H^{1} \hookrightarrow L^{q}\left(\mathbb{R}^{N}, \mathbb{R}^{2}\right)$ for all $2 \leq q \leq 2^{*}=2 N /(N-2)$.

We assume throughout the paper that $f, g: \mathbb{R}^{N} \times \mathbb{R}^{2} \rightarrow \mathbb{R}$ are nontrivial measurable functions satisfying the following hypotheses:

$$
\begin{cases}|f(x, t)| \leq C\left(|t|+|t|^{p}\right) & \text { for a.e. }(x, t) \in \mathbb{R}^{N} \times \mathbb{R}^{2} \\ |g(x, t)| \leq C\left(|t|+|t|^{p}\right) & \text { for a.e. }(x, t) \in \mathbb{R}^{N} \times \mathbb{R}^{2}\end{cases}
$$

where $p<2^{*}$;

$$
\left\{\begin{array}{l}
\lim _{\delta \rightarrow 0} \operatorname{esssup}\left\{\frac{|f(x, t)|}{|t|} ;(x, t) \in \mathbb{R}^{N} \times(-\delta,+\delta)^{2}\right\}=0 \\
\lim _{\delta \rightarrow 0} \operatorname{esssup}\left\{\frac{|g(x, t)|}{|t|} ;(x, t) \in \mathbb{R}^{N} \times(-\delta,+\delta)^{2}\right\}=0
\end{array}\right.
$$

$f$ and $g$ are chosen so that the mapping $F: \mathbb{R}^{N} \times \mathbb{R}^{2} \rightarrow \mathbb{R}$ defined by $F\left(x, t_{1}, t_{2}\right):=\int_{0}^{t_{1}} f\left(x, \tau, t_{2}\right) d \tau+\int_{0}^{t_{2}} g(x, 0, \tau) d \tau$ satisfies

$$
\left\{\begin{array}{l}
F\left(x, t_{1}, t_{2}\right)=\int_{0}^{t_{2}} g\left(x, t_{1}, \tau\right) d \tau+\int_{0}^{t_{1}} f(x, \tau, 0) d \tau \\
\text { and } F\left(x, t_{1}, t_{2}\right)=0 \text { if and only if } t_{1}=t_{2}=0
\end{array}\right.
$$


there exists a constant $\mu>2$ such that for any $x \in \mathbb{R}^{N}$

$$
\begin{aligned}
0 & \leq \mu F\left(x, t_{1}, t_{2}\right) \\
& \leq \begin{cases}t_{1} \underline{f}\left(x, t_{1}, t_{2}\right)+t_{2} \underline{g}\left(x, t_{1}, t_{2}\right) ; & t_{1}, t_{2} \in[0,+\infty), \\
t_{1} \underline{f}\left(x, t_{1}, t_{2}\right)+t_{2} \bar{g}\left(x, t_{1}, t_{2}\right) ; & t_{1} \in[0,+\infty), t_{2} \in(-\infty, 0], \\
t_{1} \bar{f}\left(x, t_{1}, t_{2}\right)+t_{2} \bar{g}\left(x, t_{1}, t_{2}\right) ; & t_{1}, t_{2} \in(-\infty, 0], \\
t_{1} \bar{f}\left(x, t_{1}, t_{2}\right)+t_{2} \underline{g}\left(x, t_{1}, t_{2}\right) ; & t_{1} \in(-\infty, 0], t_{2} \in[0,+\infty) .\end{cases}
\end{aligned}
$$

Definition 1. A function $U=\left(u_{1}, u_{2}\right) \in E$ is called solution to the problem (4) if there exists a function $W=\left(w_{1}, w_{2}\right) \in L^{2}\left(\mathbb{R}^{N}, \mathbb{R}^{2}\right)$ such that

(i) $\underline{f}\left(x, u_{1}(x), u_{2}(x)\right) \leq w_{1}(x) \leq \bar{f}\left(x, u_{1}(x), u_{2}(x)\right)$ a.e. $x$ in $\mathbb{R}^{N}$; $\underline{g}\left(x, u_{1}(x), u_{2}(x)\right) \leq w_{2}(x) \leq \bar{g}\left(x, u_{1}(x), u_{2}(x)\right)$ a.e. $x$ in $\mathbb{R}^{N}$;

(ii) $\int_{\mathbb{R}^{N}}\left(\nabla u_{1} \nabla v_{1}+\nabla u_{2} \nabla v_{2}+a(x) u_{1} v_{1}+b(x) u_{2} v_{2}\right) d x=\int_{\mathbb{R}^{N}}\left(w_{1} v_{1}+w_{2} v_{2}\right) d x$, for all $\left(v_{1}, v_{2}\right) \in E$.

Our main result is the following.

Theorem 1. Assume that conditions (5)-(8) are fulfilled. Then Problem (4) has at least a nontrivial solution in $E$.

\section{Auxiliary Results}

We first recall some basic notions from the Clarke gradient theory for locally Lipschitz functionals (see $[4,5]$ for more details). Let $X$ be a real Banach space and assume that $I: X \rightarrow \mathbb{R}$ is a locally Lipschitz functional. Then the Clarke generalized gradient is defined by

$$
\partial I(u)=\left\{\xi \in X^{*} ; I^{0}(u, v) \geq\langle\xi, v\rangle, \text { for all } v \in X\right\},
$$

where $I^{0}(u, v)$ stands for the directional derivative of $I$ at $u$ in the direction $v$, that is,

$$
I^{0}(u, v)=\limsup _{\substack{w \rightarrow u \\ \lambda \searrow 0}} \frac{I(w+\lambda v)-I(w)}{\lambda} .
$$

Let $\Omega$ be an arbitrary domain in $\mathbb{R}^{N}$. Set

$$
\begin{aligned}
E_{\Omega}=\left\{U=\left(u_{1}, u_{2}\right) \in H^{1}\left(\Omega ; \mathbb{R}^{2}\right) ;\right. \\
\\
\left.\qquad \int_{\Omega}\left(\left|\nabla u_{1}\right|^{2}+\left|\nabla u_{2}\right|^{2}+a(x) u_{1}^{2}+b(x) u_{2}^{2}\right) d x<+\infty\right\}
\end{aligned}
$$

which is endowed with the norm

$$
\|U\|_{E_{\Omega}}^{2}=\int_{\Omega}\left(\left|\nabla u_{1}\right|^{2}+\left|\nabla u_{2}\right|^{2}+a(x) u_{1}^{2}+b(x) u_{2}^{2}\right) d x .
$$

Then $E_{\Omega}$ becomes a Hilbert space. 
Lemma 1. The functional $\Psi_{\Omega}: E_{\Omega} \rightarrow \mathbb{R}, \Psi_{\Omega}(U)=\int_{\Omega} F(x, U) d x$ is locally Lipschitz on $E_{\Omega}$.

Proof. We first observe that

$$
\begin{aligned}
F(x, U)=F\left(x, u_{1}, u_{2}\right) & =\int_{0}^{u_{1}} f\left(x, \tau, u_{2}\right) d \tau+\int_{0}^{u_{2}} g(x, 0, \tau) d \tau \\
& =\int_{0}^{u_{2}} g\left(x, u_{1}, \tau\right) d \tau+\int_{0}^{u_{1}} f(x, \tau, 0) d \tau
\end{aligned}
$$

is a Carathéodory functional which is locally Lipschitz with respect to the second variable. Indeed, by (5)

$$
\begin{aligned}
\left|F\left(x, t_{1}, t\right)-F\left(x, s_{1}, t\right)\right| & =\left|\int_{s_{1}}^{t_{1}} f(x, \tau, t) d \tau\right| \leq\left|\int_{s_{1}}^{t_{1}} C\left(|\tau, t|+|\tau, t|^{p}\right) d \tau\right| \\
& \leq k\left(t_{1}, s_{1}, t\right)\left|t_{1}-s_{1}\right| .
\end{aligned}
$$

Similarly

$$
\left|F\left(x, t, t_{2}\right)-F\left(x, t, s_{2}\right)\right| \leq k\left(t_{2}, s_{2}, t\right)\left|t_{2}-s_{2}\right|
$$

Therefore

$$
\begin{aligned}
\left|F\left(x, t_{1}, t_{2}\right)-F\left(x, s_{1}, s_{2}\right)\right| & \leq\left|F\left(x, t_{1}, t_{2}\right)-F\left(x, s_{1}, t_{2}\right)\right| \\
+ & \left|F\left(x, t_{1}, s_{2}\right)-F\left(x, s_{1}, s_{2}\right)\right| \leq k(V)\left|\left(t_{2}, s_{2}\right)-\left(t_{1}, s_{1}\right)\right|,
\end{aligned}
$$

where $V$ is a neighbourhood of $\left(t_{1}, t_{2}\right),\left(s_{1}, s_{2}\right)$.

Set

$$
\chi_{1}(x)=\max \left\{u_{1}(x), v_{1}(x)\right\}, \quad \chi_{2}(x)=\max \left\{u_{2}(x), v_{2}(x)\right\} \quad \text { for all } x \in \Omega .
$$

It is obvious that if $U=\left(u_{1}, u_{2}\right), V=\left(v_{1}, v_{2}\right)$ belong to $E_{\Omega}$, then $\left(\chi_{1}, \chi_{2}\right) \in E_{\Omega}$. So, by Hölder's inequality and the continuous embedding $E_{\Omega} \subset L^{p}\left(\Omega ; \mathbb{R}^{2}\right)$,

$$
\left|\Psi_{\Omega}(U)-\Psi_{\Omega}(V)\right| \leq C\left(\left\|\chi_{1}, \chi_{2}\right\|_{E_{\Omega}}\right)\|U-V\|_{E_{\Omega}},
$$

which concludes the proof.

The following result is a generalization of Lemma 6 in [14].

Lemma 2. Let $\Omega$ be an arbitrary domain in $\mathbb{R}^{N}$ and let $f: \Omega \times \mathbb{R}^{2} \rightarrow \mathbb{R}$ be a Borel function such that $f(x,.) \in L_{\mathrm{loc}}^{\infty}\left(\mathbb{R}^{2}\right)$. Then $\underline{f}$ and $\bar{f}$ are Borel functions.

Proof. Since the requirement is local we may suppose that $f$ is bounded by $M$ and it is nonnegative. Denote by

$$
f_{m, n}\left(x, t_{1}, t_{2}\right)=\left(\int_{t_{1}-\frac{1}{n}}^{t_{1}+\frac{1}{n}} \int_{t_{2}-\frac{1}{n}}^{t_{2}+\frac{1}{n}}\left|f\left(x, s_{1}, s_{2}\right)\right|^{m} d s_{1} d s_{2}\right)^{\frac{1}{m}} .
$$


Since $\bar{f}\left(x, t_{1}, t_{2}\right)=\lim _{\delta \rightarrow 0} \operatorname{esssup}\left\{f\left(x, s_{1}, s_{2}\right) ;\left|t_{i}-s_{i}\right| \leq \delta ; i=1,2\right\}$ we deduce that for every $\varepsilon>0$, there exists $n \in \mathbb{N}^{*}$ such that for $\left|t_{i}-s_{i}\right|<\frac{1}{n}(i=1,2)$ we have $\left|\operatorname{esssup} f\left(x, s_{1}, s_{2}\right)-\bar{f}\left(x, t_{1}, t_{2}\right)\right|<\varepsilon$ or, equivalently,

$$
\bar{f}\left(x, t_{1}, t_{2}\right)-\varepsilon<\operatorname{esssup} f\left(x, s_{1}, s_{2}\right)<\bar{f}\left(x, t_{1}, t_{2}\right)+\varepsilon .
$$

By the second inequality in (9) we obtain

$$
f\left(x, s_{1}, s_{2}\right) \leq \bar{f}\left(x, t_{1}, t_{2}\right)+\varepsilon \quad \text { a.e. } x \in \Omega \quad \text { for }\left|t_{i}-s_{i}\right|<\frac{1}{n}(i=1,2)
$$

which yields

$$
f_{m, n}\left(x, t_{1}, t_{2}\right) \leq\left(\bar{f}\left(x, t_{1}, t_{2}\right)+\varepsilon\right)\left(\sqrt{4 / n^{2}}\right)^{\frac{1}{m}}
$$

Let

$$
A=\left\{\left(s_{1}, s_{2}\right) \in \mathbb{R}^{2} ;\left|t_{i}-s_{i}\right|<\frac{1}{n}(i=1,2) ; \bar{f}\left(x, t_{1}, t_{2}\right)-\varepsilon \leq f\left(x, s_{1}, s_{2}\right)\right\} .
$$

By the first inequality in (9) and the definition of the essential supremum we obtain that $|A|>0$ and

$$
f_{m, n} \leq\left(\iint_{A}\left(f\left(x, s_{1}, s_{2}\right)\right)^{m} d s_{1} d s_{2}\right)^{\frac{1}{m}} \geq\left(\bar{f}\left(x, s_{1}, s_{2}\right)-\varepsilon\right)|A|^{1 / m} .
$$

Since (10) and (11) imply

$$
\bar{f}\left(x, t_{1}, t_{2}\right)=\lim _{n \rightarrow \infty} \lim _{m \rightarrow \infty} f_{m, n}\left(x, t_{1}, t_{2}\right),
$$

it suffices to prove that $f_{m, n}$ is Borel. Let

$$
\begin{gathered}
\mathcal{M}=\left\{f: \Omega \times \mathbb{R}^{2} \rightarrow \mathbb{R} ;|f| \leq M \text { and } f \text { is a Borel function }\right\}, \\
\mathcal{N}=\left\{f \in \mathcal{M} ; f_{m, n} \text { is a Borel function }\right\} .
\end{gathered}
$$

Cf. [2, p.178], $\mathcal{M}$ is the smallest set of functions having the following properties:

(i) $\left\{f \in C\left(\Omega \times \mathbb{R}^{2} ; \mathbb{R}\right) ;|f| \leq M\right\} \subset \mathcal{M}$;

(ii) $f^{(k)} \in \mathcal{M}$ and $f^{(k)} \stackrel{k}{\rightarrow} f$ imply $f \in \mathcal{M}$.

Since $\mathcal{N}$ contains obviously the continuous functions and $(i i)$ is also true for $\mathcal{N}$ then, by the Lebesgue dominated convergence theorem, we obtain that $\mathcal{M}=N$. For $\underline{f}$ we note that $\underline{f}=-(-\bar{f}))$ and the proof of Lemma 2 is complete.

Let us now assume that $\Omega \subset \mathbb{R}^{N}$ is a bounded domain. By the continuous embedding $L^{p+1}\left(\Omega ; \mathbb{R}^{2}\right) \hookrightarrow L^{2}\left(\Omega ; \mathbb{R}^{2}\right)$, we may define the locally Lipchitz functional $\Psi_{\Omega}: L^{p+1}\left(\Omega ; \mathbb{R}^{2}\right) \rightarrow \mathbb{R}$ by $\Psi_{\Omega}(U)=\int_{\Omega} F(x, U) d x$.

Lemma 3. Under the above assumptions and for any $U \in L^{p+1}\left(\Omega ; \mathbb{R}^{2}\right)$, we have

$$
\partial \Psi_{\Omega}(U)(x) \subset[\underline{f}(x, U(x)), \bar{f}(x, U(x))] \times[\underline{g}(x, U(x)), \bar{g}(x, U(x))] \text { a.e. } x \text { in } \Omega,
$$


in the sense that if $W=\left(w_{1}, w_{2}\right) \in \partial \Psi_{\Omega}(U) \subset L^{p+1}\left(\Omega ; \mathbb{R}^{2}\right)$ then

$$
\begin{aligned}
& \underline{f}(x, U(x)) \leq w_{1}(x) \leq \bar{f}(x, U(x)) \quad \text { a.e. } x \text { in } \Omega, \\
& \underline{g}(x, U(x)) \leq w_{2}(x) \leq \bar{g}(x, U(x)) \quad \text { a.e. } x \text { in } \Omega .
\end{aligned}
$$

Proof. By the definition of the Clarke gradient we have

$$
\int_{\Omega}\left(w_{1} v_{1}+w_{2} v_{2}\right) d x \leq \Psi_{\Omega}^{0}(U, V) \quad \text { for all } V=\left(v_{1}, v_{2}\right) \in L^{p+1}\left(\Omega ; \mathbb{R}^{2}\right) .
$$

Choose $V=(v, 0)$ such that $v \in L^{p+1}(\Omega), v \geq 0$ a.e. in $\Omega$. Thus, by Lemma 2 ,

$$
\begin{aligned}
\int_{\Omega} w_{1} v & \leq \limsup _{\substack{\left(h_{1}, h_{2}\right) \rightarrow U \\
\lambda \backslash 0}} \frac{\int_{\Omega}\left(\int_{h_{1}(x)}^{h_{1}(x)+\lambda v(x)} f\left(x, \tau, h_{2}(x)\right) d x\right.}{\lambda} \\
& \leq \int_{\Omega}\left(\limsup _{\substack{\left(h_{1}, h_{2}\right) \rightarrow U \\
\lambda \backslash 0}} \frac{1}{\lambda} \int_{h_{1}(x)}^{h_{1}(x)+\lambda v(x)} f\left(x, \tau, h_{2}(x)\right) d x\right. \\
& \leq \int_{\Omega} \bar{f}\left(x, u_{1}(x), u_{2}(x)\right) v(x) d x .
\end{aligned}
$$

Analogously we obtain

$$
\int_{\Omega} \underline{f}\left(x, u_{1}(x), u_{2}(x)\right) v(x) d x \leq \int_{\Omega} w_{1} v d x \quad \text { for all } v \geq 0 \text { in } \Omega .
$$

Arguing by contradiction, suppose that (12) is false. Then there exist $\varepsilon>0$, a set $A \subset \Omega$ with $|A|>0$ and $w_{1}$ as above such that

$$
w_{1}(x)>\bar{f}(x, U(x))+\varepsilon \quad \text { in } A .
$$

Taking $v=\mathbf{1}_{A}$ in (14) we obtain

$$
\int_{\Omega} w_{1} v d x=\int_{A} w_{1} d x \leq \int_{A} \bar{f}(x, U(x)) d x,
$$

which contradicts (16). Proceeding in the same way we obtain the corresponding result for $g$ in (13).

Define $\Psi_{\Omega}: E_{\Omega} \rightarrow \mathbb{R}, \Psi_{\Omega}(U)=\int_{\Omega} F(x, U) d x$. With the same arguments as in the proof of Lemma 3 and using the embedding $E_{\Omega} \hookrightarrow L^{p+1}\left(\Omega, \mathbb{R}^{2}\right)$, we obtain

$$
\partial \Psi_{\Omega}(U)(x) \subset[\underline{f}(x, U(x)), \bar{f}(x, U(x))] \times[\underline{g}(x, U(x)), \bar{g}(x, U(x))] \quad \text { a.e. } x \in \Omega .
$$

Let $V \in E_{\Omega}$. Then $\tilde{V} \in E$, where $\tilde{V}: \mathbb{R}^{N} \rightarrow \mathbb{R}^{2}$ is defined by

$$
\tilde{V}= \begin{cases}V(x), & x \text { in } \Omega \\ 0 & \text { otherwise }\end{cases}
$$


For $W \in E^{*}$ we consider $W_{\Omega} \in E_{\Omega}^{*}$ such that $\left\langle W_{\Omega}, V\right\rangle=\langle W, \tilde{V}\rangle$ for all $V$ in $E_{\Omega}$. Set $\Psi: E \rightarrow \mathbb{R}, \Psi(U)=\int_{\mathbb{R}^{N}} F(x, U)$.

Lemma 4. Let $W \in \partial \Psi(U)$, where $U \in E$. Then $W_{\Omega} \in \partial \Psi_{\Omega}(U)$, in the sense that $W_{\Omega} \in \partial \Psi_{\Omega}\left(\left.U\right|_{\Omega}\right)$.

Proof. By the definition of the Clarke gradient we deduce that $\langle W, \tilde{V}\rangle \leq$ $\Psi^{0}(U, \tilde{V})$ for all $V$ in $E_{\Omega}$

$$
\begin{aligned}
\Psi^{0}(U, \tilde{V}) & =\limsup _{\substack{H \rightarrow U, H \in E \\
\lambda \rightarrow 0}} \frac{\Psi(H+\lambda \tilde{V})-\Psi(H)}{\lambda} \\
& =\limsup _{\substack{H \rightarrow U, H \in E \\
\lambda \rightarrow 0}} \frac{\int_{\mathbb{R}^{N}}(F(x, H+\lambda \tilde{V})-F(x, H)) d x}{\lambda} \\
& =\limsup _{\substack{H \rightarrow U, H \in E \\
\lambda \rightarrow 0}} \frac{\int_{\Omega}(F(x, H+\lambda \tilde{V})-F(x, H)) d x}{\lambda} \\
& =\limsup _{\substack{H \rightarrow U, H \in E_{\Omega} \\
\lambda \rightarrow 0}} \frac{\int_{\Omega}(F(x, H+\lambda \tilde{V})-F(x, H)) d x}{\lambda}=\Psi_{\Omega}^{0}(U, V) .
\end{aligned}
$$

Hence $\left\langle W_{\Omega}, V\right\rangle \leq \Psi_{\Omega}^{0}(U, V)$ which implies $W_{\Omega} \in \partial \Psi_{\Omega}^{0}(U)$.

By Lemmas 3 and 4 we obtain that for any $W \in \partial \Psi(U)$ (with $U \in E$ ), $W_{\Omega}$ satisfies (12) and (13). We also observe that for $\Omega_{1}, \Omega_{2} \subset \mathbb{R}^{N}$ we have $\left.W_{\Omega_{1}}\right|_{\Omega_{1} \cap \Omega_{2}}=\left.W_{\Omega_{2}}\right|_{\Omega_{1} \cap \Omega_{2}}$.

Let $W_{0}: \mathbb{R}^{N} \rightarrow \mathbb{R}$, where $W_{0}(x)=W_{\Omega}(x)$ if $x \in \Omega$. Then $W_{0}$ is well defined and

$$
W_{0}(x) \in[\underline{f}(x, U(x)), \bar{f}(x, U(x))] \times[\underline{g}(x, U(x)), \bar{g}(x, U(x))] \quad \text { a.e. } x \in \mathbb{R}^{N}
$$

and, for all $\varphi \in C_{c}^{\infty}\left(\mathbb{R}^{N}, \mathbb{R}^{2}\right),\langle W, \varphi\rangle=\int_{\mathbb{R}^{N}} W_{0} \varphi$. By density of $C_{c}^{\infty}\left(\mathbb{R}^{N}, \mathbb{R}^{2}\right)$ in $E$ we deduce that $\langle W, V\rangle=\int_{\mathbb{R}^{N}} W_{0} V d x$ for all $V$ in $E$. Hence

$$
\begin{aligned}
W(x)=W_{0}(x) \in[\underline{f}(x, U(x)), \bar{f}(x, U(x))] & \\
& \times[\underline{g}(x, U(x)), \bar{g}(x, U(x))] \quad \text { a.e. } x \in \mathbb{R}^{N} .
\end{aligned}
$$

\section{Proof of Theorem 1}

Define the energy functional $I: E \rightarrow \mathbb{R}$

$$
\begin{aligned}
I(U) & =\frac{1}{2} \int_{\mathbb{R}^{N}}\left(\left|\nabla u_{1}\right|^{2}+\left|\nabla u_{2}\right|^{2}+a(x) u_{1}^{2}+b(x) u_{2}^{2}\right) d x-\int_{\mathbb{R}^{N}} F(x, U) d x \\
& =\frac{1}{2}\|U\|_{E}^{2}-\Psi(U) .
\end{aligned}
$$


The existence of solutions to problem (4) will be justified by a nonsmooth variant of the Mountain-Pass Theorem (see Chang [3] and Rădulescu [17]) applied to the functional $I$, even if the $P S$ condition is not fulfilled. More precisely, we check the following geometric hypotheses:

$$
I(0)=0 \quad \text { and there exists } V \in E \quad \text { such that } I(V) \leq 0 \text {; }
$$

there exist $\beta, \rho>0 \quad$ such that $I \geq \beta \quad$ on $\left\{U \in E ;\|U\|_{E}=\rho\right\}$.

VERIFICATION OF (19). It is obvious that $I(0)=0$. For the second assertion we need the following lemma.

Lemma 5. There exist two positive constants $C_{1}$ and $C_{2}$ such that

$$
f(x, s, 0) \geq C_{1} s^{\mu-1}-C_{2} \quad \text { for a.e. } x \in \mathbb{R}^{N} ; s \in[0,+\infty),
$$

where the constant $\mu$ has been defined in (8).

Proof. We first observe that (8) implies

$$
0 \leq \mu F(x, s, 0) \leq \begin{cases}s f(x, s, 0) & s \in[0,+\infty) \\ s \overline{\bar{f}}(x, s, 0) & s \in(-\infty, 0]\end{cases}
$$

which places us in the conditions of Lemma 5 in [14].

Verification of (19) COntinued. Choose $v \in C_{c}^{\infty}\left(\mathbb{R}^{N}\right)-\{0\}$ so that $v \geq 0$ in $\mathbb{R}^{N}$. We have $\int_{\mathbb{R}^{N}}|\nabla v|^{2}+a(x) v^{2}<\infty$, hence $t(v, 0) \in E$ for all $t \in \mathbb{R}$. Thus by Lemma 5 we obtain

$$
\begin{aligned}
I(t(v, 0)) & =\frac{t^{2}}{2} \int_{\mathbb{R}^{N}}|\nabla v|^{2}+a(x) v^{2} d x-\int_{\mathbb{R}^{N}} \int_{0}^{t v} f(x, \tau, 0) d \tau \\
& \leq \frac{t^{2}}{2} \int_{\mathbb{R}^{N}}|\nabla v|^{2}+a(x) v^{2} d x-\int_{\mathbb{R}^{N}} \int_{0}^{t v}\left(C_{1} \tau^{\mu-1}-C_{2}\right) d \tau \\
& =\frac{t^{2}}{2} \int_{\mathbb{R}^{N}}|\nabla v|^{2}+a(x) v^{2} d x+C_{2} t \int_{\mathbb{R}^{N}} v d x-C_{1}^{\prime} t^{\mu} \int_{\mathbb{R}^{N}} v^{\mu} d x<0
\end{aligned}
$$

for $t>0$ large enough.

VERIFICATION OF (20). We observe that (6), (7) and (8) imply that, for any $\varepsilon>0$, there exists a constant $A_{\varepsilon}>0$ such that

$$
\begin{aligned}
& |f(x, s)| \leq \varepsilon|s|+A_{\varepsilon}|s|^{p}, \\
& |g(x, s)| \leq \varepsilon|s|+A_{\varepsilon}|s|^{p}
\end{aligned} \quad \text { for a.e. }(x, s) \in \mathbb{R}^{N} \times \mathbb{R}^{2} .
$$


By (21) and Sobolev's embedding theorem we have, for any $U \in E$,

$$
\begin{aligned}
|\Psi(U)|= & \left|\Psi\left(u_{1}, u_{2}\right)\right| \leq \int_{\mathbb{R}^{N}} \int_{0}^{\left|u_{1}\right|} \| f\left(x, \tau, u_{2}\right)\left|d \tau+\int_{\mathbb{R}^{N}} \int_{0}^{u_{2}}\right| g(x, 0, \tau) \mid d \tau \\
\leq & \int_{\mathbb{R}^{N}}\left(\frac{\varepsilon}{2}\left|\left(u_{1}, u_{2}\right)\right|^{2}+\frac{A_{\varepsilon}}{p+1} \mid\left(u_{1},\left.u_{2}\right|^{p+1}\right) d x\right. \\
& +\int_{\mathbb{R}^{N}}\left(\frac{\varepsilon}{2}\left|u_{2}\right|^{2}+\frac{A_{\varepsilon}}{p+1}\left|u_{2}\right|^{p+1}\right) d x \\
\leq & \varepsilon\|\|_{L^{2}}^{2}+\frac{2 A_{\varepsilon}}{p+1}\|U\|_{L^{p+1}}^{p+1} \leq \varepsilon C_{3}\|U\|_{E}^{2}+C_{4}\|U\|_{E}^{p+1},
\end{aligned}
$$

where $\varepsilon$ is arbitrary and $C_{4}=C_{4}(\varepsilon)$. Thus

$$
I(U)=\frac{1}{2}\|U\|_{E}^{2}-\Psi(U) \geq \frac{1}{2}\|U\|_{E}^{2}-\varepsilon C_{3}\|U\|_{E}^{2}-C_{4}\|U\|_{E}^{p+1} \geq \beta>0,
$$

for $\|U\|_{E}=\rho$, with $\rho, \varepsilon$ and $\beta$ sufficiently small positive constants.

Denote

$$
\mathcal{P}=\{\gamma \in C([0,1], E) ; \gamma(0)=0, \gamma(1) \neq 0 \quad \text { and } I(\gamma(1)) \leq 0\}
$$

and

$$
c=\inf _{\gamma \in \mathcal{P}} \max _{t \in[0,1]} I(\gamma(t)) .
$$

Set

$$
\lambda_{I}(U)=\min _{\xi \in \partial I(U)}\|\xi\|_{E^{*}} .
$$

Thus, by the nonsmooth version of the Mountain Pass Lemma [3], there exists a sequence $\left\{U_{M}\right\} \subset E$ such that

$$
I\left(U_{m}\right) \rightarrow c \quad \text { and } \quad \lambda_{I}\left(U_{m}\right) \rightarrow 0 .
$$

So, there exists a sequence $\left\{W_{m}\right\} \subset \partial \Psi\left(U_{m}\right) ; W_{m}=\left(w_{m}^{1}, w_{m}^{2}\right)$ such that

$$
\left(-\Delta u_{m}^{1}+a(x) u_{m}^{1}-w_{m}^{1},-\Delta u_{m}^{2}+a(x) u_{m}^{2}-w_{m}^{2}\right) \rightarrow 0 \quad \text { in } E^{*} .
$$

Note that, by (8),

$$
\begin{gathered}
\Psi(U) \leq \frac{1}{\mu}\left(\int_{u_{1} \geq 0} u_{1}(x) \underline{f}(x, U) d x+\int_{u_{1} \leq 0} u_{1}(x) \bar{f}(x, U) d x\right. \\
\left.+\int_{u_{2} \geq 0} u_{1}(x) \underline{g}(x, U) d x+\int_{u_{2} \leq 0} u_{2}(x) \bar{g}(x, U) d x\right) .
\end{gathered}
$$

Therefore, by (17),

$$
\Psi(U) \leq \frac{1}{\mu} \int_{\mathbb{R}^{N}} U(x) W(x) d x=\frac{1}{\mu} \int_{\mathbb{R}^{N}}\left(u_{1} w_{1}+u_{2} w_{2}\right) d x,
$$


for every $U \in E$ and $W \in \partial \Psi(U)$. Hence, if $\langle\cdot, \cdot\rangle$ denotes the duality pairing between $E^{*}$ and $E$, we have

$$
\begin{aligned}
I\left(U_{m}\right)= & \frac{\mu-2}{2 \mu} \int_{\mathbb{R}^{N}}\left(\left|\nabla u_{m}^{1}\right|^{2}+\left|\nabla u_{m}\right|^{2}+a(x)\left|u_{m}\right|^{1}+b(x)\left|u_{m}\right|^{2}\right) d x \\
& +\frac{1}{\mu}\left\langle\left(-\Delta u_{m}^{1}+a(x) u_{m}^{1}-w_{m}^{1},-\Delta u_{m}^{2}+b(x) u_{m}^{2}-w_{m}^{2}\right), U_{m}\right\rangle \\
& +\frac{1}{\mu}\left\langle W_{m}, U_{m}\right\rangle-\Psi\left(U_{m}\right) \\
\geq & \frac{\mu-2}{2 \mu} \int_{\mathbb{R}^{N}}\left(\left|\nabla u_{m}^{1}\right|^{2}+\left|\nabla u_{m}^{2}\right|^{2}+a(x)\left|u_{m}^{1}\right|^{2}+b(x)\left|u_{m}^{2}\right|^{2}\right) d x \\
& +\frac{1}{\mu}\left\langle\left(-\Delta u_{m}^{1}+a(x) u_{m}^{1}-w_{m}^{1},-\Delta u_{m}^{2}+b(x) u_{m}^{2}-w_{m}^{2}\right), U_{m}\right\rangle \\
\geq & \frac{\mu-2}{2 \mu}\left\|U_{m}\right\|_{E}^{2}-o(1)\left\|U_{m}\right\|_{E} .
\end{aligned}
$$

This relation in conjunction with (22) implies that the Palais-Smale sequence $\left\{U_{m}\right\}$ is bounded in $E$. Thus, it converges weakly (up to a subsequence) in $E$ and strongly in $L_{\text {loc }}^{2}\left(\mathbb{R}^{N}\right)$ to some $U$. Taking into account that $W_{m} \in \partial \Psi\left(U_{m}\right)$ and $U_{m} \rightarrow U$ in $E$, we deduce from (23) that there exists $W \in E^{*}$ such that $W_{m} \rightarrow W$ in $E^{*}$ (up to a subsequence). Since the mapping $U \longmapsto F(x, U)$ ia compact from $E$ to $L^{1}$, it follows that $W \in \partial \Psi(U)$. Therefore

$$
W(x) \in[\underline{f}(x, U(x)), \bar{f}(x, U(x))] \times[\underline{g}(x, U(x)), \bar{g}(x, U(x))] \quad \text { a.e. } x \in \mathbb{R}^{N}
$$

and

$$
\begin{gathered}
\left(-\Delta u_{m}^{1}+a(x) u_{m}^{1}-w_{m}^{1},-\Delta u_{m}^{2}+b(x) u_{m}^{2}-w_{m}^{2}\right)=0 \\
\Longleftrightarrow \int_{\mathbb{R}^{N}}\left(\nabla u_{1} \nabla v_{1}+\nabla u_{2} \nabla v_{2}+a(x) u_{1} v_{1}+b(x) u_{2} v_{2}\right) d x \\
=\int_{\mathbb{R}^{N}}\left(w_{1} v_{1}+w_{2} v_{2}\right) d x \text { for all }\left(v_{1}, v_{2}\right) \in E .
\end{gathered}
$$

These last two relations show that $U$ is a solution pf the problem (4).

It remains to prove that $U \not \equiv 0$. If $\left\{W_{m}\right\}$ is as in (23) then, by relations (22), (24) and for large $m$

$$
\begin{aligned}
\frac{c}{2} & \leq I\left(U_{m}\right)-\frac{1}{2}\left\langle\left(-\Delta u_{m}^{1}+a(x) u_{m}^{1}-w_{m}^{1},-\Delta u_{m}^{2}+b(x) u_{m}^{2}-w_{m}^{2}\right), U_{m}\right\rangle \\
& =\frac{1}{2}\left\langle W_{m}, U_{m}\right\rangle-\int_{\mathbb{R}^{N}} F\left(x, U_{m}\right) d x=\frac{1}{2}\left\langle W_{m}, U_{m}\right\rangle-\Psi\left(x, U_{m}\right) \\
& \leq \frac{1}{2}\left(\int_{u_{1} \geq 0} u_{1}(x) \underline{f}(x, U) d x+\int_{u_{1} \leq 0} u_{1}(x) \bar{f}(x, U) d x\right.
\end{aligned}
$$




$$
\left.+\int_{u_{2} \geq 0} u_{1}(x) \underline{g}(x, U) d x+\int_{u_{2} \leq 0} u_{2}(x) \bar{g}(x, U) d x\right) .
$$

Now, taking into account the definition of $\bar{f}, \underline{f}, \bar{g}, \underline{g}$ we deduce that $\bar{f}, \underline{f}, \bar{g}, \underline{g}$ verify (19), too. So by (25) we obtain

$$
\frac{c}{2} \leq \int_{\mathbb{R}^{N}}\left(\varepsilon\left|U_{m}\right|^{2}+A_{\varepsilon}\left|u_{m}\right|^{p+1}\right)=\varepsilon\left\|U_{m}\right\|_{L^{2}}^{2}+A_{\varepsilon}\left\|U_{m}\right\|_{L^{p+1}}^{p+1} .
$$

So, $\left\{U_{m}\right\}$ does not converge strongly to 0 in $L^{p+1}\left(\mathbb{R}^{N} ; \mathbb{R}^{2}\right)$. From now on, a standard argument implies that $U \not \equiv 0$, which concludes our proof.

\section{ACKNOWLEDGEMENTS}

The author is grateful to the anonymous referee for the careful reading of the manuscript and for numerous suggestions that substantially improved the initial version of this paper.

\section{REFERENCES}

1. A. Ambrosetti and P. H. Rabinowitz, Dual variational methods in critical point theory and applications. J. Functional Analysis 14(1973), 349-381.

2. S. K. Berberian, Measure and integration. The Macmillan Co., New York; CollierMacmillan Ltd., London, 1965.

3. K. C. Chang, Variational methods for nondifferentiable functionals and their applications to partial differential equations. J. Math. Anal. Appl. 80(1981), No. 1, 102-129.

4. F. H. Clarke, Generalized gradients and applications. Trans. Amer. Math. Soc. 205(1975), 247-262.

5. F. H. Clarke, Generalized gradients of Lipschitz functionals. Adv. in Math. 40(1981), No. 1, 52-67.

6. B. D. Esry, C. H. Greene, J. P. Burke, Jr., and J. L. Bohn, Hartree-Fock theory for double condensates. Phys. Rev. Lett. 78(1997), 3594-3597.

7. F. Gazzola and V. RĂdulescu, A nonsmooth critical point theory approach to some nonlinear elliptic equations in $\mathbb{R}^{n}$. Differential Integral Equations 13(2000), No. 1-3, 4760 .

8. D. Gilbarg and N. S. Trudinger, Elliptic partial differential equations of second order. Second edition. Grundlehren der Mathematischen Wissenschaften [Fundamental Principles of Mathematical Sciences], 224. Springer-Verlag, Berlin, 1983.

9. H. Grosse and A. Martin, Particle physics and the Schrödinger equation. Cambridge Monographs on Particle Physics, Nuclear Physics and Cosmology, 6. Cambridge University Press, Cambridge, 1997.

10. D. H. Hyers, G. Isac, and T. M. Rassias, Topics in nonlinear analysis \& applications. World Scientific Publishing Co., Inc., River Edge, NJ, 1997.

11. A. Hasegawa and Y. Kodama, Solitons in Optical Communications. Oxford Series in Optical and Imaging Sciences. 7. Clarendon Press., Oxford, 1995.

12. M. N. Islam, Ultrafast fiber switching devices and systems. Cambridge University Press, New York, 1992. 
13. T. Kato, Remarks on holomorphic families of Schrödinger and Dirac operators. Differential equations (Birmingham, Ala., 1983), 341-352, North-Holland Math. Stud., 92, North-Holland, Amsterdam, 1984.

14. P. Mironescu and V. Rădulescu, A multiplicity theorem for locally Lipschitz periodic functionals. J. Math. Anal. Appl. 195(1995), No. 3, 621-637.

15. Y.-G. OH, Existence of semiclassical bound states of nonlinear Schrödinger equations with potentials of the class $(V)_{a}$. Comm. Partial Differential Equations 13(1988), No. 12, 1499-1519.

16. P. H. Rabinowitz, On a class of nonlinear Schrödinger equations. Z. Angew. Math. Phys. 43(1992), No. 2, 270-291.

17. V. D. RĂDUlESCU, Mountain pass theorems for nondifferentiable functions and applications. Proc. Japan Acad. Ser. A Math. Sci. 69(1993), No. 6, 193-198.

18. C. Sulem and P.-L. Sulem, The nonlinear Schrödinger equation. Self-focusing and wave collapse. Applied Mathematical Sciences, 139. Springer-Verlag, New York, 1999.

(Received 8.08.2005)

Author's address:

Department of Mathematics

"Fratii Buzeşti" College

Bd. Ştirbei-Vodă No. 5, 200352 Craiova

Romania

E-mail: tldinu@gmail.com 